\title{
Mechanical and material properties of the subchondral bone plate from the femoral head of patients with osteoarthritis or osteoporosis
}

\author{
Baohua Li, Richard M Aspden
}

\begin{abstract}
Objective-To determine the material properties of the subchondral bone plate in patients with osteoarthritis or osteoporosis.

Methods-Femoral heads were obtained after surgical removal from age and sex matched groups of patients with either osteoporosis (OP), after a fractured neck of femur, or osteoarthritis (OA) and compared with a normal group. The mechanical stiffness, density, and composition of the subchondral bone plate from sites selected to represent areas of heavy, intermittent, and light loading were measured. Results-Overall, OP bone was the least stiff and dense, followed by OA bone; normal bone was stiffer and more dense $(p<$ 0.05). Though OP bone contained less mineral, the organic and water contents were increased in proportion suggesting no change in the relative amount of organic matrix. OA bone was also hypomineralised $(p<0.05)$ but had different organic and water fractions suggesting a defect in the matrix. Site variation of most properties was small, though across all the groups the superior region was significantly stiffer than the inferior.

Conclusion-This study shows that subchondral bone plate is less stiff than normal in both OP and OA and so cannot, by itself, explain the preserving of the overlying cartilage in OP while aiding its destruction in OA. However, the subchondral bone plate is only one part of the bony structure of the femoral head and changes in the cancellous bone need to be considered. The generalised changes in bone composition found in patients with OA support the hypothesis that the disease could involve the bone in the primary pathogenesis.
\end{abstract}

(Ann Rheum Dis 1997;56:247-254)

Department of Orthopaedic Surgery, University of Aberdeen, Scotland $\mathrm{B} \mathrm{Li}$

R M Aspden

Correspondence to: Dr R M Aspden, Department of

Orthopaedics, University of Aberdeen, Polwarth Building, Foresterhill, Aberdeen AB9 2ZD.

Accepted for publication 9 December 1996 the western world. They are among the greatest causes of morbidity in the elderly ${ }^{1}$ and place an enormous demand on orthopaedic
This study investigates the mechanical and changes occur in the properties of the bone in these patients. OA and OP together afflict a services; about one quarter of all orthopaedic beds in the United Kingdom are occupied by patients who have sustained a fracture of the femoral neck resulting from OP and a further quarter by patients electing for a joint replacement for OA. Clinical studies have shown that these diseases are rarely present together in the same patient and that presence of one may be protective against the other. ${ }^{23}$ Byers $e t$ al $l^{4}$ noted that the progressive changes of OA were not seen in over 100 femoral heads removed after fracture of the femoral neck. In a study of a random population sample in Jerusalem, Pogrund $e t a l^{5}$ found that the prevalence of OP and OA in 641 pelvic $x$ rays separately was $16.1 \%$ and $4.1 \%$, but in only $0.5 \%$ did $\mathrm{OA}$ and $\mathrm{OP}$ coexist in the same person. Epidemiological surveys have suggested a negative association between the two conditions ${ }^{6}$ and supported the hypothesis that these are two distinct diseases and not phenomena related solely to aging. However, it has not been established whether this apparent lack of association occurs by chance or if there are underlying causal factors.

The pathogeneses of both OA and OP are still not known. OA affects all the major joint tissues, including the synovium, articular cartilage, subchondral bone, and cancellous bone. It is generally regarded as a disease of cartilage, the bony changes being thought to be secondary to this, and most research and treatment is focused on trying prevent or reduce the degeneration of the articular cartilage. OP, however, is universally considered to be a disease of bone, though the reasons for the loss of bone and the inability of the cells to regulate properly the mechanical properties of the tissue are not fully understood. Part of the reason for our lack of understanding of both of these diseases is because of the difficulties in recognising early disease and obtaining human tissue for study at this stage.

Though it is widely held that OA is a primary disorder of articular cartilage with secondary change in the bone, it has been suggested that the health and integrity of the overlying articular cartilage depend on the mechanical properties of its bony bed. ${ }^{78} \mathrm{~A}$ defect in the subchondral bone, resulting in a reduction of its compliance, may result in greater stresses being sustained by the articular cartilage leading to overloading and consequent breakdown. Conversely, an increase in compliance, such as may be expected due to the loss of bone in OP, may have a protective effect. Previous studies of subchondral bone in 
Table 1 Details of groups from which femoral heads were removed. Significance values for sex and age matching are from $\chi^{2}$ and non-parametric ANOVA tests respectively enabling acceptance of the null hypothesis of no difference between groups

\begin{tabular}{llll}
\hline Group & $\begin{array}{l}\text { Number of } \\
\text { femoral heads }\end{array}$ & $\begin{array}{l}\text { Sex } \\
\text { (female/male) }\end{array}$ & $\begin{array}{l}\text { Age } \\
\text { (median(youngest, oldest)) }\end{array}$ \\
\hline Osteoporotic & 17 & $13 / 4$ & $77(66,91)$ \\
Osteoarthritic & 16 & $12 / 4$ & $73(49,86)$ \\
Normal & 7 & $5 / 2$ & $79(58,93)$ \\
& & $\mathrm{p}=0.97$ & $\mathrm{p}=0.44$ \\
\hline
\end{tabular}

relation to its possible role in the pathogenesis of OA have concentrated on morphological findings ${ }^{9-12}$ and we have not found any investigation into the mechanical properties and composition of the subchondral bone plate other than a study of the hardness of subchondral bone in the patella. ${ }^{13}$ This study investigates this question by measuring the stiffness, the density, and the composition of the subchondral bone plate itself and compares the results obtained from patients with OA or OP with a normal group to discover the importance of changes in the subchondral bone to the clinical presentation of OA and OP.

\section{Methods}

Samples of subchondral bone were taken from femoral heads collected from three clinical groups, OA, OP, and normal, which were matched for age and sex. The femoral heads from both disease groups were obtained in the operating theatre from patients undergoing a hip replacement for either a fractured neck of femur attributed to OP or for OA of the hip. Cases with roentgenographic, biochemical, or histological evidence of osteomalacia, multiple myeloma, rheumatoid arthritis, or secondary osteoporosis due to corticosteroids were excluded from the osteoporotic group. Patients with rheumatoid arthritis, osteomalacia, congenital or acquired dysplasia, gout, or avascular necrosis were excluded from the osteoarthritic group. A control group was collected from hips removed during postmortem examination and the medical records were examined to exclude known disorders affecting bone metabolism. A clinical definition of each

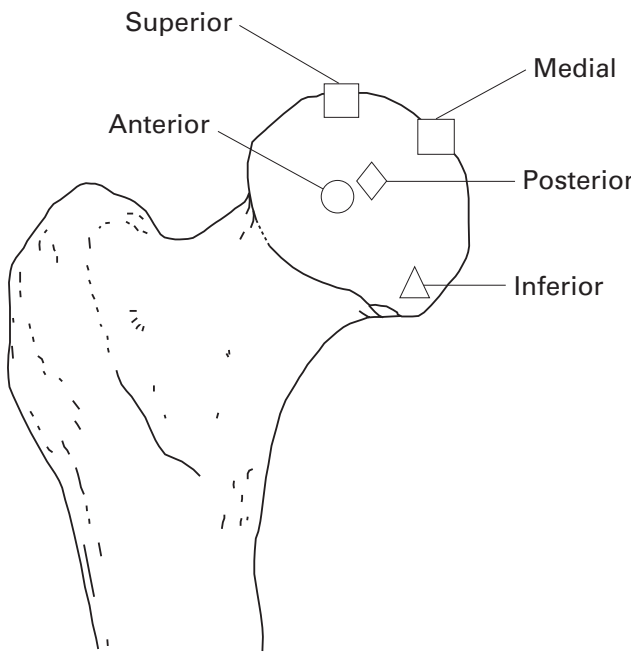

Figure1 Schematic diagram of the sites on the human femoral head from which samples of subchondral bone were removed.

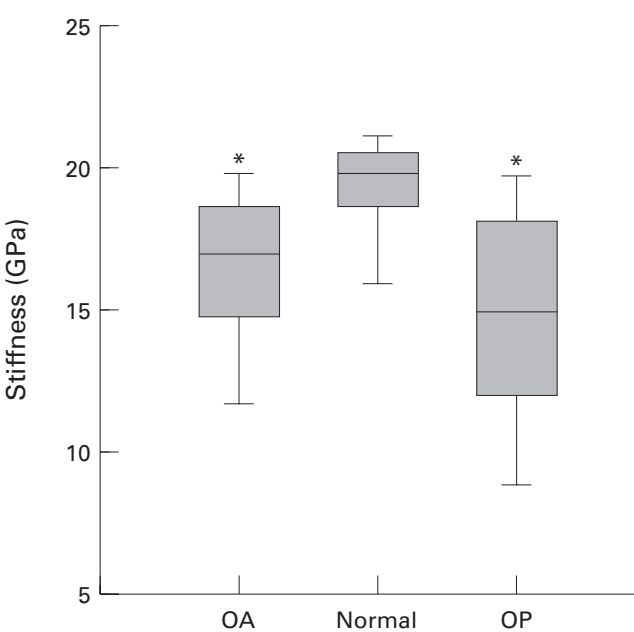

Figure 2 Median values of the stiffness of the subchondral bone plate determined from all sites over the femoral head of patients undergoing a hip replacement for osteoarthritis $(O A)$, or osteoporosis $(O P)$ compared with normal controls. The extent of the shaded bars shows the $25 \%$ and $75 \%$ confidence limits, the error bars show the 5\% and $95 \%$ limits. $\left({ }^{\star} p<0.05\right)$.

disease was used to determine what overall changes occur in the bone of patients judged suitable for surgery as National Health Service patients. No attempt was made to grade the severity of disease. Table 1 shows the median ages and the oldest and youngest of each of the patient groups. The ratio of female to male was not significantly different in any group and there was no difference in the age distributions between the groups. All samples were stored at $4^{\circ} \mathrm{C}$ in a calcium phosphate buffered saline solution containing sodium azide as this has been shown to preserve the structure and composition of the bone. ${ }^{14}$

From each femoral head, cylindrical cores of $9 \mathrm{~mm}$ diameter were removed from sites chosen to represent regions subjected to different amounts of loading in vivo (fig 1): superior being the most heavily loaded; posterior, anterior and medial from the partially loaded region; and inferior being the least loaded. ${ }^{15} 16$ Care was taken to maintain the bone at all times in a fully hydrated condition. Any articular cartilage on the surface of the specimen was removed with a scalpel. The subchondral bone was cut from each core using a rotary electric saw (Struers Accutom-2) fitted with a silicon carbide cut off wheel rotating at $300 \mathrm{rpm}$, cooled with distilled water. This resulted in a disc of bone less than $1 \mathrm{~mm}$ thick. In preparation for ultrasonic measurement of the stiffness, the remains of the trabeculae and the cartilage were removed and the surfaces made parallel and polished by sequential grinding on a series of graded silicon carbide grinding papers, finishing with 4000 grit paper. The specimens were subsequently cleaned in an ultrasonic bath filled with calcium-phosphate buffered saline solution to remove any traces of silicon carbide. The thickness of each specimen, $d$, was measured five times using a micrometer and the mean used in subsequent calculations.

An ultrasonic method was used to determine the elastic stiffness modulus of the bone. 


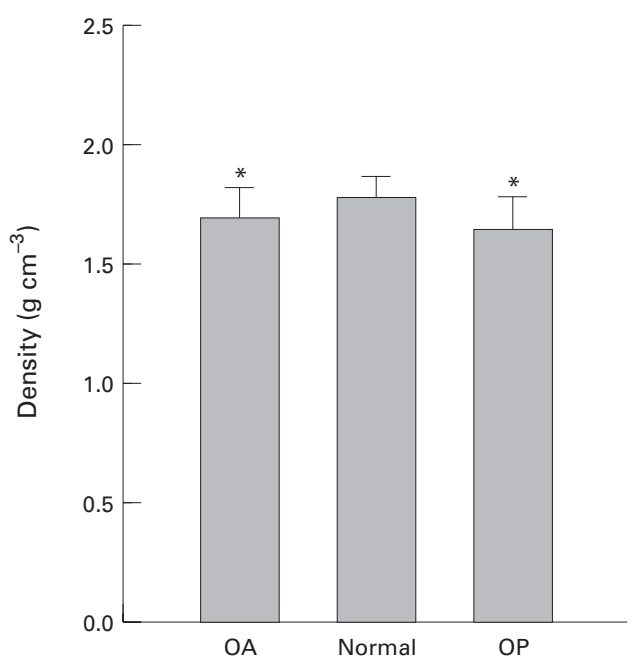

Figure 3 Density of the subchondral bone plate from the femoral heads of patients with $O A$ or OP compared with normal controls. Both $O A$ and OP groups are significantly reduced from normal and $O P$ is slightly but significantly less than $\mathrm{OA}$. $\left({ }^{\star} p<0.05\right)$.

Ultrasound, of $10 \mathrm{MHz}$ frequency, was obtained using a pulsar/receiver (Model 5052 PR, Panametrics Inc) and a straight beam contact transducer (Panametrics Inc). The specimen was firmly pressed onto the wear plate of the transducer with a drop of distilled water to ensure good acoustic coupling and the transit time, $\mathrm{T}$, was measured using a single transmitter/receiver and a dual beam oscilloscope (Hitachi V-665A, Japan) by the pulse-echo method. The cartilage surface of the bone was always placed adjacent to the transducer. The longitudinal sonic plesiovelocity was calculated using $\mathrm{v}_{\ell}=2 \mathrm{~d} / \mathrm{T}$. The
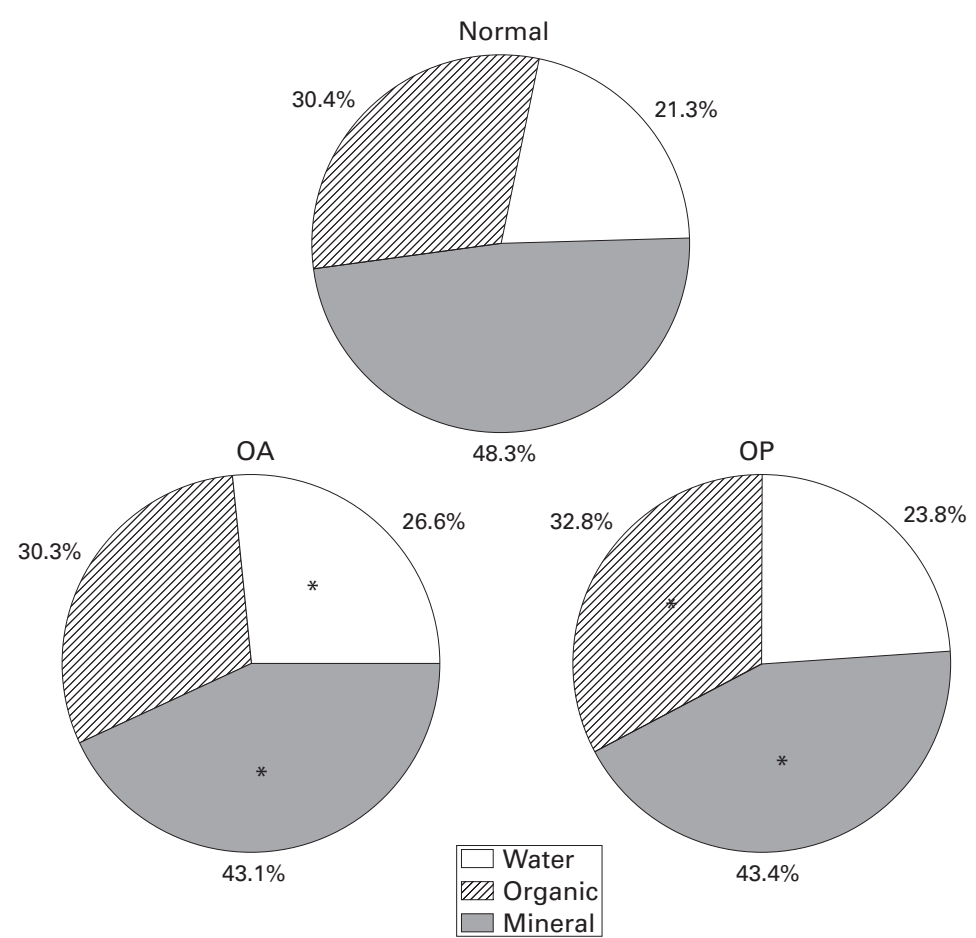

Figure 4 Composition of normal, $O A$, and $O P$ subchondral bone plate from human femoral heads expressed as a fraction of the wet mass of the sample. Both OP and OA differed from normal but in different ways. $\left({ }^{\star} p<0.05\right)$. effective path length for sound in an anisotropic, inhomogeneous medium, such as bone, is not known, and will generally be longer than the specimen. The estimation of longitudinal velocity will, therefore, probably be an underestimate and has been termed the plesio-velocity for this reason. ${ }^{17}$

The density of each specimen, $\rho$, was determined using Archimedes' principle by weighing it in air, after removing excess water by gentle patting with a damp tissue, and then suspended in calcium phosphate buffer solution of known density. Each measurement was repeated five times and the mean determined. The longitudinal elastic stiffness modulus was calculated from the formula $\mathrm{K}=\rho \mathrm{v}_{\ell}^{2} \cdot{ }^{17}$

To determine the composition of the bone, the specimens were dehydrated at $105^{\circ} \mathrm{C}$ for 24 hours and weighed again. The water content is the difference between the wet weight and the dry weight. They were then ashed at $600^{\circ} \mathrm{C}$ for 24 hours and reweighed to determine the ash weight, which was taken to be the mineral content. The organic content was calculated by subtracting the ash weight from the dry weight. The mass of each component was then expressed as a fraction of the wet mass of the sample and also as mass per unit volume of tissue by multiplying the mass fraction by the density of the specimen, as determined above.

The results were analysed first to determine differences in the overall results in each patient group and then to investigate site variation within each group. Because these may be interrelated a two way analysis of variance (ANOVA) was performed. Normality of the distributions was assessed using the Kolmogorov-Smirnov test with the significance level, p, set at 0.05 . Mean values and associated standard deviations are shown for those data that are normally distributed, otherwise median values and associated 5\%,25\%, $75 \%$, and $95 \%$ intervals are shown. Pairwise comparisons were made using StudentNewman-Keuls method for comparing all groups or Dunnett's method for comparison with a control. Unconstrained linear regression was used to determine trends and possible relations between variables.

\section{Results}

THICKNESS AND APPEARANCE

The mean (SD) thickness of the prepared subchondral bone plate specimens in each of the patient groups was $0.69(0.19) \mathrm{mm}$ in the OA group $(n=73), 0.60(0.11) \mathrm{mm}$ in the normal group $(\mathrm{n}=32)$, and $0.56(0.17) \mathrm{mm}$ in the OP group $(n=74)$. Only the OA specimens were significantly different from normal $(p<0.05)$ and there was no significant variation with site or interaction between site and disease group as shown by two way ANOVA. Despite being thicker the OA bone was difficult to prepare as it was coarse in texture, crumbled easily, and the boundary with the underlying cancellous bone was indistinct. OP subchondral bone was thinner but appeared qualitatively stronger. In all groups, removal and preparation of an intact specimen from 


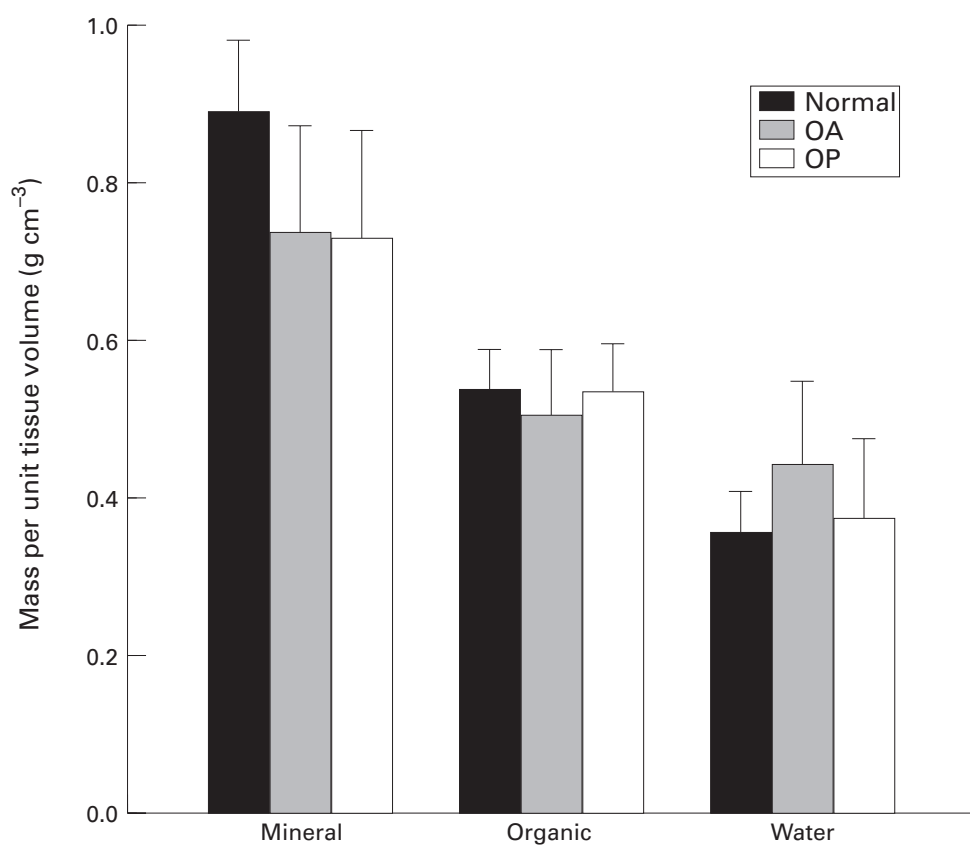

Figure 5 Composition of normal, $O A$, and OP subchondral bone plate expressed as mass per unit volume of tissue showing the changes seen in figure 4 in a different way. Both the $O A$ and OP bone contain less mineral than normal $(p<0.05)$, though they have the same organic content. The increased water content in the OA bone matrix $(p<0.05$ compared with normal) suggests that matrix changes occurring in the $O A$ bone are different to those in OP bone. is denoted by $\mathrm{n}$. Both the stiffness and the density are significantly lower $(\mathrm{p}<0.05)$ in the OA and the OP groups than in the normal group and in neither case was there any significant interaction between disease group and site as shown by two way ANOVA. This also showed that across all the disease groups the stiffness of the superior region was significantly greater than that of the inferior $(\mathrm{p}<0.05)$ though there was no corresponding variation in density.

COMPOSITION

The compositions of the OA and the OP groups both differed from the normal group but in different ways (fig 4). The OA samples had a reduced mass fraction of mineral and an increased mass fraction of water compared with normal, both of which were significant $(p<0.05)$. The OP samples also had a significantly smaller mass fraction of mineral compared with normal but the organic and water contents were both greater than normal, though only the organic component significantly $(\mathrm{p}<0.05)$. Two way ANOVA showed that there was no significant interaction between the disease group and the site for any of the composition variables. Multiplying the mass fraction by the tissue density yields a measure of the composition as mass per unit volume (fig 5). From this it may be seen that OP subchondral bone has organic and water contents that are similar to normal, simply a reduction in the amount of mineral $(\mathrm{p}<0.05)$. In contrast the OA bone is not only hypomineralised compared with normal $(p<0.05)$ but there is an increased water content $(\mathrm{p}<0.05)$ though no change in organic content.

Figure 2 shows the median stiffness and figure 3 the density of subchondral bone from all sites in each of the three patient groups. The stiffness (19.8 MPa) and density (mean (SD)) $\left(1.79(0.08) \mathrm{g} \mathrm{cm}^{-3}, \mathrm{n}=29\right)$ were both greatest in the normal group, those in the OP group were lowest $\left(14.9 \mathrm{MPa}\right.$ and $1.64(0.13) \mathrm{g} \mathrm{cm}^{-3}$, $\mathrm{n}=65$ ) and the OA group lay in between though nearer to the OP than to the normals (17.0 MPa and $\left.1.69(0.13) \mathrm{g} \mathrm{cm}^{-3}, \mathrm{n}=67\right)$. The number of samples removed intact and tested

RELATION BETWEEN STIFFNESS AND DENSITY

Density is one of the main determining factors of bone stiffness and a low but significant correlation was found between the stiffness and density in all three groups (fig 6). The equations of the regression lines and the adjusted correlation coefficients were:

Normal: $\mathrm{K}=-10.5+16.6 \rho\left(\mathrm{R}_{\text {adj }}^{2}=0.24, \mathrm{p}=\right.$ $0.004)$

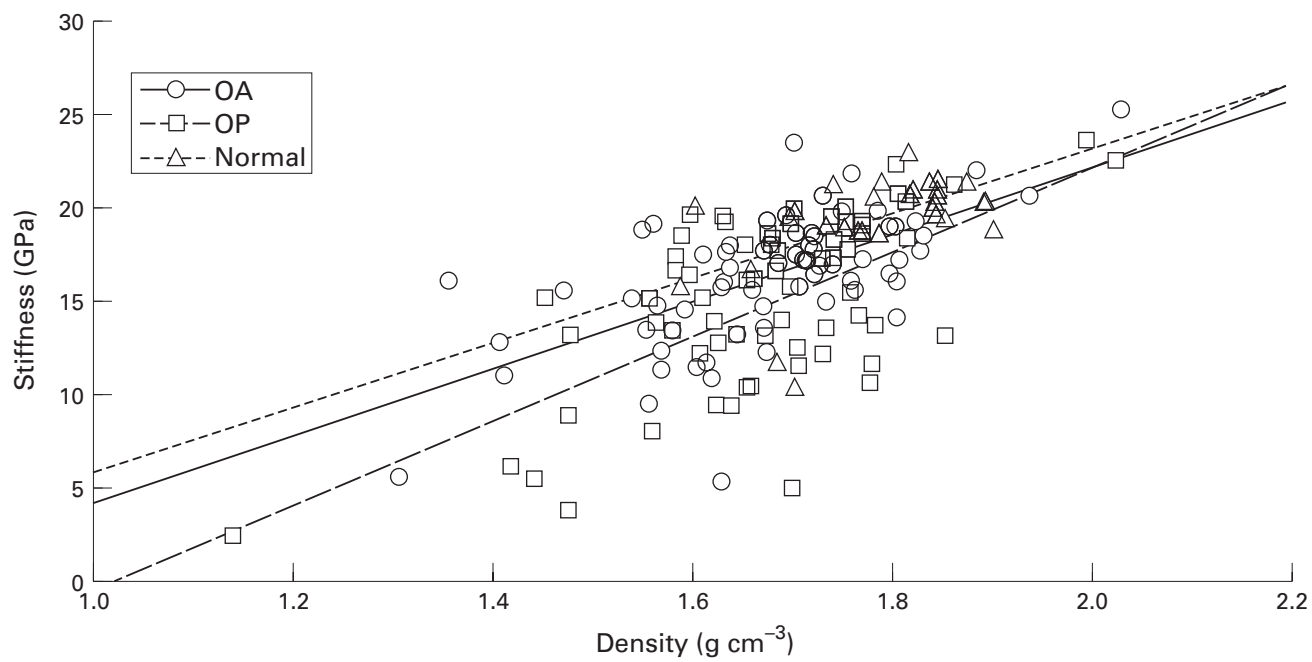

Figure 6 Linear regression relations between stiffness and density of the subchondral bone plate. There is no difference between the gradients of the regression lines over the density range found in this study. See text for regression equations. 
$\mathrm{OA}: \mathrm{K}=-13.1+17.4 \rho\left(\mathrm{R}_{\text {adj }}^{2}=0.39, \mathrm{p}<\right.$ $0.0001)$

$\mathrm{OP}: \mathrm{K}=-21.4+22.1 \rho\left(\mathrm{R}_{\text {adj }}^{2}=0.40, \mathrm{p}<\right.$ $0.0001)$

A Student's $t$ test showed that there was no significant difference between these slopes ( $p>0.5$ for all comparisons). Only in the OA and OP groups was there a significant correla-
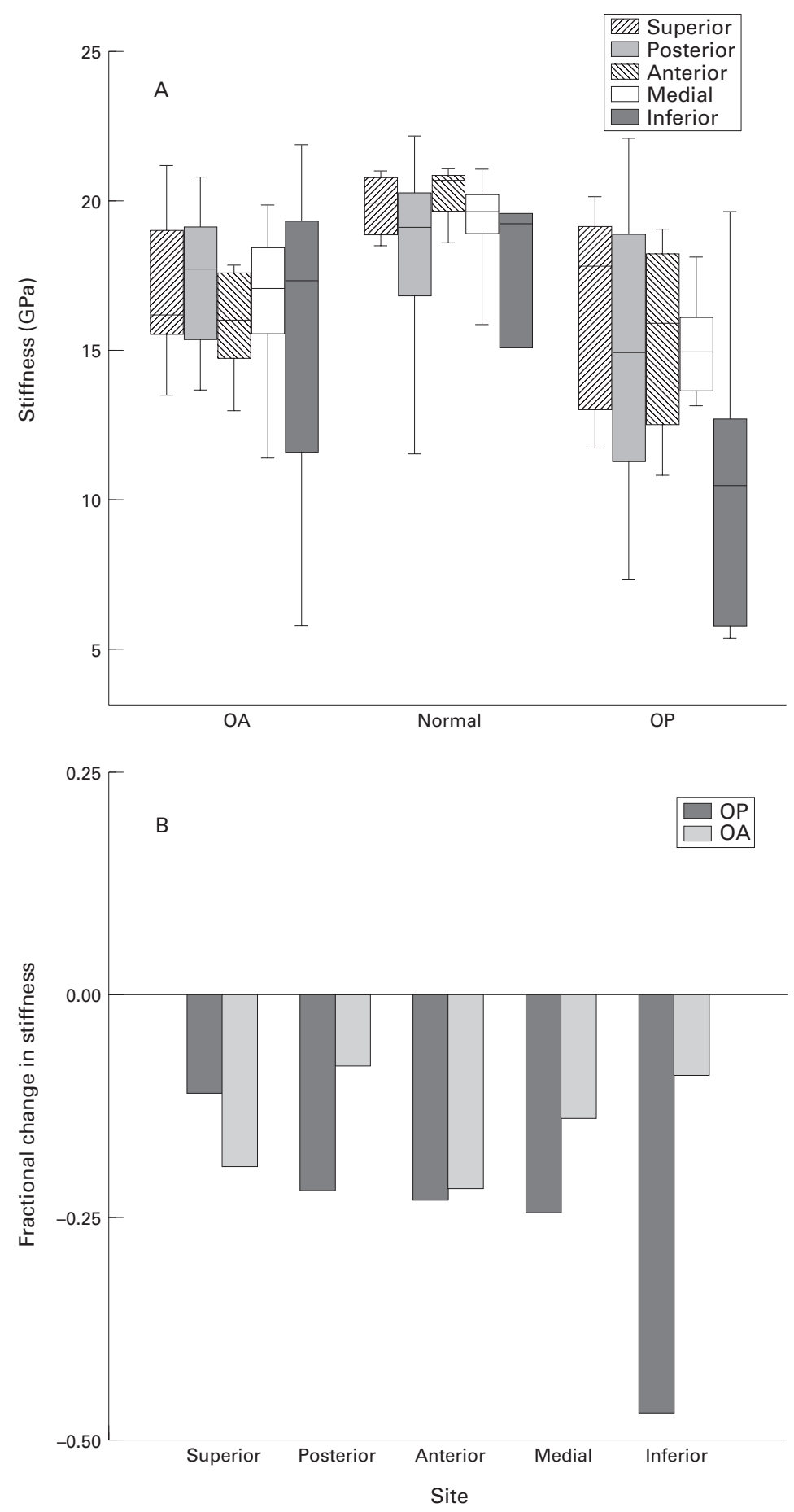

Figure 7 Site variation of stiffness of the subchondral bone plate from human femoral heads. (A) Stiffnesses are not normally distributed and the median values are shown. Shaded bars represent $25 \%$ and $75 \%$ and the errors bars the $5 \%$ and $95 \%$ confidence intervals. (B) Stiffness of each site expressed as a fractional change compared with the equivalent site from the normal group. tion between sonic velocity and density though the correlation coefficients $(\mathrm{R})$ were only 0.34 and 0.50 respectively.

SITE VARIATION

There was very little site variation in any of the parameters measured (fig 7(A)). A two way ANOVA showed that for all disease groups the stiffness of the bone from the inferior region was less than that from the superior $(p<0.05)$. A more detailed analysis using one way ANOVA within each group showed that there was no site variation in any group apart from the OP group in which the inferior was significantly $(p<0.05)$ less than the superior. Expressing these as a fractional change compared with the values obtained from the corresponding site in the normal tissue, using the formula (diseased-normal)/normal, showed that at nearly all sites from both $\mathrm{OA}$ and $\mathrm{OP}$ groups there was a reduction in the stiffness of the subchondral bone (fig 7(B)) and only in the inferior and posterior regions was this noticeably greater in the OP than in the OA group. These results were reflected in the density measurements where there was not found any significant site variation (fig 8(A)) and the fractional changes were marked only in the anterior and inferior regions, especially in the OP group (fig $8(\mathrm{~B})$ ). No significant variation was found in the composition measurements from the various sites.

\section{Discussion}

This study shows that changes occur in the subchondral bone plate immediately adjacent to the articular cartilage in the femoral heads of patients with $\mathrm{OA}$ or OP. Of greatest significance is that the stiffness of the bone from patients with either OA or OP is less than that from a non-clinical group but that the causes of this reduction are different in each group. The stiffness of the osteoporotic bone was reduced by $22 \%$ and its density by about $8 \%$ while the stiffness of the osteoarthritic bone was $14 \%$ less than that of normal and the density was reduced by $6 \%$. Though both patient groups showed similar reductions in the amount of mineral, which being the most dense is the main component that would account for these changes, the relative amounts of organic material and water were different.

There was very little site variation in any of the disease groups though numbers in some of the groups were small, for instance only four in the normal inferior group, which may be why some of the measured changes did not reach significance. Other studies have suggested a correlation between the thickness of the subchondral bone plate and the prevailing mechanical load ${ }^{18}$ and it may be that the tissue responds to load by regulating the amount of bone rather than the material properties of the bone. We did not measure the thickness of the subchondral bone plate as both surfaces are irregular and the thickness can only be measured properly by cutting vertical sections. This was not compatible with having uniform, circular, parallel faced specimens for ultrasound measurement.

Obtaining normal tissue for postmortem examination is difficult and there will always be 
doubt in a study like this as to how 'normal' are the normal group. Because of the requirement for matching by age and sex, the tissue was obtained from an elderly population, which could easily have contained people with sub-clinical OA or OP. We excluded any femoral heads with marked degeneration of the articular cartilage but were not able to collect samples in the same number as can be obtained from the operating theatre. Using a clinical definition of disease means that we can
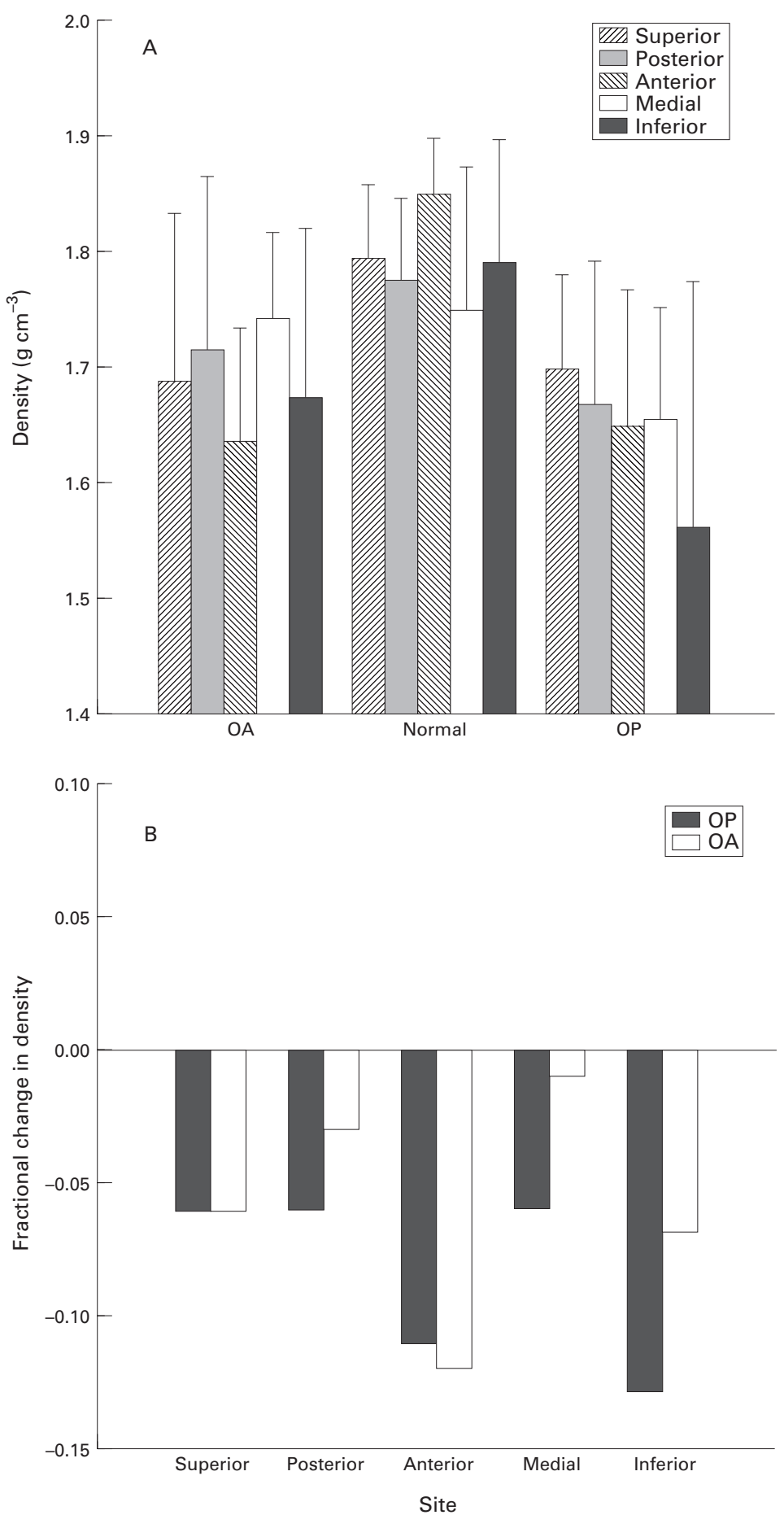

Figure 8 (A) Site variation of density of the subchondral bone plate from human femoral heads. (B) Density of each site expressed as a fractional change compared with the equivalent site from the normal group. define the 'normal' group to be clinically normal though we cannot be sure that there is no overlap in the material properties of the groups. However, it is essential to have some group to which the disease groups can be compared otherwise it is not possible to determine whether and in which ways they have deviated from normal. The self consistency of the results from this and our other studies ${ }^{19}$ gives us reasonable confidence that what we have measured are real changes in the bone. Clearly both the OA and the OP samples are from the end stages of the disease, as shown by their removal at surgery, and this was deemed sufficient to be able to identify the significant changes that have taken place. Further studies could look for changes during the course of the disease by comparison with an appropriate scoring system but this would require many more samples to have sufficient stages represented. In addition neither the Mankin score for OA cartilage nor the Singh index for OP bone have a scientific basis and provide only a qualitative description of some of the observable changes, indeed the Singh index has been shown to be unreliable as a radiological indicator of bone content in femoral heads. ${ }^{20}$ The specimens were prepared so that highly polished, parallel faces were produced, and these were checked visually. Scanning electron microscopy shows that there is little or no calcified cartilage in the OA samples and the appearance of the OP and normal specimens strongly suggested that little of the softer calcified cartilage remained after grinding and polishing.

Ultrasound has successfully been used before to measure the elastic stiffness modulus of bone. ${ }^{171-25}$ Ultrasonic measurement offers certain advantages in that it measures the elastic properties of the material; viscoelasticity and its associated time dependency is effectively removed due to the high frequencies involved. It is also non-destructive, although this second factor also means that it cannot provide information on yield properties or ultimate strength. Successive demineralisation of bone pieces has shown that the longitudinal sonic plesio-velocity increases linearly with density. ${ }^{22}$ However, it is also affected by the anisotropy and porosity of the bone. ${ }^{21}$ The subchondral bone plate in the femoral head is a compact calcified tissue, with a typical thickness of less than a millimetre, which makes difficult the processing of samples for physical measurements. It is certainly impossible to cut a piece of suitable size and regular shape for testing with a conventional materials testing machine and, perhaps because of this, we are aware of only two other studies investigating the physical properties of subchondral bone plate, ${ }^{1326}$ and those both used an indentation technique to measure the hardness. The results of one study showed a reduction in the hardness of the medial tibial plateau in both $\mathrm{OA}$ and rheumatoid arthritis ${ }^{26}$ while the other found no appreciable difference between normal and OA bone in the human patella, though no statistical comparison was presented. In our experiments, obtaining 
suitable samples, especially from the inferior region, was not always possible and the sample numbers and the larger errors shown in the site variation of stiffness are a consequence of this. We also found a poor correlation between velocity and density, which suggests that there are changes in the structure of the bone and not in the mineral content alone.

The changes in mass fractions of the major components of the bone clearly show that there are changes in composition with disease. However, these results are not easy to interpret because, when expressed as a fraction, a change in only one component would alter the apparent values of the other components. For instance, it is readily calculated that if there is no change in the absolute amounts of organic and water but the mineral mass fraction is reduced from $48.3 \%$ to $43.4 \%$ then the mass fractions of organic and water will appear to increase to $33.3 \%$ and $23.3 \%$ respectively, which are almost identical with the values we measured from the osteoporotic bone. Further insight into what is occurring may be gained by expressing the content of each component as the mass per unit volume. These data (fig 5) further support the result of this calculation, that there is no absolute change in the water and organic contents, and suggests that in the OP group there is a loss of mineralisation of the bone. Whether this is due to hypomineralisation of the matrix or an increase in the amount of unmineralised osteoid needs to be investigated.

In contrast, changes in the composition of the OA bone are more complicated. Not only is there a reduction in mineral content but there are also significant changes in the relative amounts of water and organic material. In a previous study ${ }^{19}$ we have found similar changes in the material density and composition of cancellous bone from all sites over the femoral head and neck and, contrary to expectation, the most heavily loaded regions had the lowest material density even though the apparent density (reflecting the amount of bone) was greatest. These results confirm another study, ${ }^{27}$ which used a density fractionation technique on finely ground bone and showed that OA subchondral bone is less dense, though in this case 'subchondral' was a $1 \mathrm{~cm}$ deep core from the surface and must have comprised mainly cancellous bone. This method produced a histogram with binned values of densities that were not related directly to the composition. Our study extends these findings by measuring the stiffness and composition as well as the exact density of the bone thereby enabling the relations between these parameters to be determined. Mineralisation has been shown to have an effect on the packing fraction of collagen fibrils; increased mineralisation leading to closer packing of the collagen molecules and a reduced water content. ${ }^{1428}$ Our results from the $\mathrm{OA}$ bone are consistent with a defect in the mineralisation of the collagen resulting in a reduced mineralisation and a decreased packing fraction. This would result in an increased water content and could be the source of at least a part of the reduction in mechanical stiffness.

The results of other studies have shown that subchondral bone becomes thickened in OA in both humans and in many animal models. ${ }^{9102729}$ Our results support these findings but with the considerable reservation that we measured the thickness of the samples only after preparation for ultrasonic stiffness measurements. We took care to remove only the minimum amount of bone compatible with obtaining smooth parallel faces, so our results will be a reflection of the thickness but not an accurate measurement of it. This thickening has been related to the reduction in the ability of the OA subchondral bone to absorb energy $^{730}$ which, it is suggested, could in turn lead to breakdown of the cartilage. It has always been assumed that the bone material itself is normal. This study clearly shows, however, that the bone is abnormal; exhibiting a loss of stiffness allied to a change in composition. Taken together, a thickening of more compliant bone ought, in principle, to result in an increased ability to absorb energy - thick, soft cushions are better shock absorbers than thin, hard ones. Changes in the subchondral bone plate alone then would not appear to provide a mechanism for provoking the mechanical breakdown of the articular cartilage. However, it is important not to consider the subchondral bone plate in isolation. We have shown elsewhere ${ }^{19}$ that, though similar changes are found in the composition of the underlying cancellous bone, the proliferation of bone leads to a considerable increase in stiffness of the bulk material. Energy absorption will occur not only in the subchondral bone plate but also in the underlying cancellous bone, so it may be the overall increase in stiffness that is a factor in the degeneration of the articular cartilage. In addition, the changes in the cancellous bone were found at all sites over the femoral head and neck suggesting that defective bone is not a phenomenon localised to the joint surface under an osteoarthritic lesion. Proliferation of defective bone, both subchondral and cancellous, throughout the joint could be symptomatic of a more generalised disease of the bone. ${ }^{6}$

We thank the Medical Research Council for a Senior Fellowship for RMA and the Sir Halley Stewart Trust and the Arthritis and Rheumatism Council for financial support.

1 Department of Health. On the state of public health 1993. London: HMSO, 1994;

2 Solomon L, Schnitzler CM, Browett JP. Osteoarthritis of the hip: the patient behind the disease. Ann Rheum Dis 1982; hip: the patien.

3 Cooper C, Cook PL, Osmond C, Cawley MID. Osteoarthritis of the hip and osteoporosis of the proximal femur. Ann Rheum Dis 1991;50:540-2.

4 Byers PD, Contepenni CA, Farkas TA. A post mortem study of the hip joint. Ann Rheum Dis 1970;29:15-31.

5 Pogrund H, Rutemberg M, Makin M, Robin G, Menczel J, Steiberg R. Osteoarthritis of the hip joint and osteoporosis: a radiological study in a random population sample in Jerusalem. Clin Orthop 1982;164:130-5.

6 Dequeker J, Johnell O. Osteoathritis protects against femoral neck fracture: the MEDOS study experience. Bone 1995;14:S51-6.

7 Radin EL, Paul IL. Does cartilage compliance reduce skeletal impact loads? The relative force-attenuating properties of articular cartilage, synovial fluid, periarticular soft tissues and bone. Arthritis Rheum 1970;13:139-44. 
8 Radin EL, Rose RM. The role of subchondral bone in the initiation and progression of cartilage damage. Clin Orthop 198

9 Amir G, Pirie CJ, Rashad S, Revell PA. Remodelling of subchondral bone in osteoarthritis: a histomorphometric study. J Clin Pathol 1992;45:990-2.

10 Oettmeier R, Arokoski J, Roth AJ, Helminen HJ, Tammi M, Abendroth K. Quantitative study of articular cartilage and subchondral bone remodelling in the knee joint of dogs after strenuous running training. J Bone Miner Res 1992; 7:S419-24

11 Havdrup T, Hulth A, Telhag $\mathrm{H}$. The subchondral bone in osteoarthritis and rheumatoid arthritis of the knee. A histological and microradiographical study. Acta Orthop Scand. 1976;47:345-50.

12 Benske J, Schünke M, Tillmann B. Subchondral bone formation in in arthrosis. Polychrome labelling studies in mice. Acta Orthop Scand 1988;59:536-41.

13 Björkström S, Goldie IF. Hardness of the subchondral bone of the patella in the normal state, in chondromalacia and in of the patella in the normal state, in chondromalacia
osteoarthrosis. Acta Orthop Scand 1982;53:451-62.

14 Lees S. Sonic velocity and the ultrastructure of mineralised tissues. In: Hukins DWL, ed.Calcified tissues. London, Macmillan, 1988:121-52.

15 Thomas DB, Daniel TS. In vitro contact stress distributions in the natural human hip. J Biomech. 1983;16:373-84.

16 Hodge WH, Fijan RS, Carlson KL, Burgess RG, Harri WH, Mann RW. Contact pressures in the human hip joint measured in vivo. Proc Natl Acad Sci USA 1986;83. 2879-83.

17 Lees S, Heeley JD, Cleary PF. A study of some properties of a sample of bovine cortical bone using ultrasound. Calcif Tissue Int 1979;29:107-17.

18 Armstrong SJ, Read RA, Price R. Topographical variation within the articular cartilage and subchondral bone of the normal ovine knee joint: a histological approach. Osteoarnormal ovine knee joint: a histo

$19 \mathrm{Li} \mathrm{B}$, Aspden RM. Composition and mechanical properties of cancellous bone from the femoral head of patients with osteoporosis or osteoarthritis. J Bone Miner Res (in press).
20 Wicks $M$. Absence of metabolic bone disease in the proximal femur in patients with fracture of the femoral neck. J Bone Joint Surg 1982;64-B:319-22.

21 Lees S, Ahern JM, Leonard M. Parameters influencing the sonic velocity in compact calcified tissues of various species. J Acoust Soc Am 1983;74:28-33.

22 Tavakoli MB, Evans JA. Dependence of the velocity and attenuation of ultrasound in bone on the mineral content. Phys Med Biol 1991;36:1529-37.

23 Turner $\mathrm{CH}$, Eich $\mathrm{M}$. Ultrasonic velocity as a predictor of strength in bovine cancellous bone. Calcif Tissue Int 1991;49:116-9.

24 Yoon HS, Katz JL. Ultrasonic wave propagation in human cortical bone II: measurements of elastic properties and microhardness. J Biomech 1976;9:459-64.

25 Lang SB. Ultrasonic method for measuring elastic coefficients of bone and results on fresh and dried bovine bone. IEEE Trans Biomed Eng 1970;17:101-5.

26 Lereim P, Goldie I, Dalberg E. Hardness of the subchondral bone of the tibial condyles in the normal state and in osteoarthritis and rheumatoid arthritis. Acta Orthop Scand 1974;45:614-27.

27 Grynpas MD, Alpert B, Katz I, Lieberman I, Pritzker KPH. Subchondral bone in osteoarthritis. Calcif Tissue Int 1991;49:20-6.

28 Lees S. Water content in type I collagen tissues calculated from the generalized packing model. Int J Biol Macromol 1986;8:66-72.

29 Dedrick DK, Goldstein SA, Brandt KD, O'Connor BL, Goulet RW, Albrecht M. A longitudinal study of subchondral plate and trabecular bone in cruciate deficient dogs with osteoarthritis followed up for 54 months. Arthritis Rheum 1993;36:1460-7.

30 Simon SR, Radin EL, Paul IL, Rose RM. The response of joints to impact loading. II in vivo behaviour of subchondral bone. J Biomech 1972;5:267-72. 